\title{
Spectroscopic Characteristics of Xeloda Chemodrug
}

\author{
Sahar Abdollahi Jahdi ${ }^{\circledR}$, Parviz Parvin $^{1 *}{ }^{\circledR}$, Solaleh Seyedi ${ }^{1}$, Saeid Jelvani ${ }^{\circledR},{ }^{\circledR}$ Mohammad Reza \\ Razzaghi $^{3}$
}

1Physics Department, Amirkabir University of Technology, Tehran, Iran

${ }^{2}$ Photonics and Quantum Technologies Research School, Nuclear Science and Technology Research Institute (NSTRI), Tehran, Iran

${ }^{3}$ Laser Application in Medical Sciences Research Center, Shahid Beheshti University of Medical Sciences, Tehran, Iran

\section{*Correspondence to Parviz Parvin, Email: parvin@ aut.ac.ir}

Received: December 28, 2020 Accepted: February 2, 2021 Published online September 25 2021

\begin{abstract}
Introduction: Spectroscopic properties of Xeloda chemodrug have been studied over varying concentrations ranging between 0.001 and $10 \mathrm{mg} / \mathrm{mL}$, using laser-induced fluorescence (LIF) spectroscopy. The alternative photoluminescence (PL) and near infrared (NIR) measurements are carried out to authenticate the obtained results by the LIF method. Methods: The $\mathrm{XeCl}$ laser as the excitation coherent source with $160 \mathrm{~mJ} /$ pulse at $308 \mathrm{~nm}$ is employed for LIF measurements of the fluorophore of interest in the modular spectroscopic set-up. Results: Xeloda as a significant chemodrug acts as a notable fluorophore. LIF, PL and NIR spectroscopy techniques are employed to investigate the spectral properties of the chemodrug in terms of concentration. The maximum LIF peak intensity of Xeloda is achieved at $\lambda_{\max }=410.5 \mathrm{~nm}$ and the characteristic concentration of $C_{\mathrm{P} 1}=0.05 \mathrm{mg} / \mathrm{mL}$. PL signals are in good agreement with the data given by the LIF measurements. The characteristic NIR spectra of Xeloda as solid evidence of chemical bonding formation attest to fluorescence quenching at the fluorophore concentration of $0.2 \mathrm{mg} /$ $\mathrm{mL}$. Besides, the spectral shift of fluorescence signals which is obtained in terms of fluorophore concentration- demonstrating as a diagnostic marker for the purpose of optimized chemotherapy. Conclusion: Xeloda exhibits outstanding fluorescence properties over the allowable concentration in human serum $\left(\mathrm{C}_{\max }\right)$. These characteristics could benefit potential advantage of simultaneous laserbased imaging of cell-chemodrug interaction over in-vivo studies.

Keywords: Xeloda; Spectroscopy; LIF; PL; Spectral shift.
\end{abstract}

\section{Introduction}

Surgery and radiation therapy are used to remove, kill or damage cancer cells in a certain area, whereas chemotherapy acts throughout the whole body to not only palliate but also cure the disease. Xeloda (Xeloda, Roche) is used as the oral prodrug of fluorouracil (FU) in second-line palliative chemotherapy for different metastatic cancers such as colon, gastric and breast with the aim of increasing tolerability and selectivity of tumor cells. ${ }^{1,2}$ Xeloda is administrated as an oral form of FU + leucovorin (LV) combination with superior efficacy and safety profile improvement. ${ }^{3}$ This chemodrug as an alternative to fluorouracil is also combined with other drugs such as oxaliplatin in diverse chemotherapy regimens for the treatment of different stages of cancer. ${ }^{4}$ There is a rapid growth in demand for fast optical spectroscopic measurements in biomedical applications. On the other hand, laser-based measurements such as Raman spectroscopy and laser-induced breakdown spectroscopy find an effective contribution in cancer diagnostic methods and cellular imaging. ${ }^{5-9}$ However, the laser-induced fluorescence (LIF) technique enjoys several privileges against other diagnosis techniques relying on exogenous/endogenous biocompatible fluorophores which promote the measurements to a more controllable and highly selective level. ${ }^{9-11}$ Besides, the advantages of simultaneous imaging alongside photothermal therapy and chemotherapy could be achieved by the fluorescent chemodrugs. ${ }^{12-15}$

Recently, the fluorescent properties of Xeloda (Capecitabine) have been studied using the ArF laser at a high concentration range of $0.25-10 \mathrm{mg} / \mathrm{mL} .{ }^{16}$ Here, the $\mathrm{XeCl}$ laser as an alternative adaptable source in the clinical approach was exploited to excite Xeloda fluorophore. Moreover, LIF spectroscopy of Xeloda was carried out over varying concentrations ranging from 0.001 to $10 \mathrm{mg} / \mathrm{mL}$ to characterize its fluorescent properties in favor of low and high intake doses. The validation 
of LIF spectroscopy of Xeloda was examined under photoluminescence (PL) measurements. Furthermore, near infrared (NIR) spectra were also recorded in various concentrations to investigate the correlation of chemical bindings and fluorescence emissions. To the best of our knowledge, there is no similar report in the literature to investigate the fluorescence properties of Xeloda based on the comparative spectroscopic approach.

\section{Materials and Methods}

Xeloda (Roche $\mathrm{GmbH}$ ) with $\mathrm{MW}=359.354 \mathrm{~g} / \mathrm{mol}$ was solved in deionized water and exposed to ultrasonic irradiation to prepare a homogeneous and welldissolved solution. UV-VIS spectroscopy of Xeloda was implemented by the Cary 60 spectro-photometer with 1.5 nm optical resolution over 190-1100 nm. Furthermore, the optical behavior of Xeloda solutions was studied utilizing PL and LIF techniques over a wide range of these chemodrug concentrations ranging from 0.001 to 10 $\mathrm{mg} / \mathrm{mL}$. Moreover, PL and LIF spectra were achieved by averaging over at least 50 measurements for more reliable assessments.

LIF measurements were performed by making use of the $\mathrm{XeCl}$ laser at the center wavelength of $308 \mathrm{~nm}, 160$ $\mathrm{mJ} /$ pulse, $20 \mathrm{~ns}$ pulse duration and the repetition rate of $10 \mathrm{~Hz}$. The laser beam was focused on the solution using a focusing lens with $8 \mathrm{~cm}$ focal length. Table 1 represents the laser beam properties such as type of laser, emission

Table 1. XeCl Laser Parameters

\begin{tabular}{ll}
\hline Type of laser: $\mathrm{XeCl}$ Laser & Spot diameter at the focus: $500 \mu \mathrm{m}-2 \mathrm{~mm}$ \\
\hline Emission mode: Pulse & Focus spot area: $2-6 \mathrm{~mm}^{2}$ \\
\hline Delivery system: Direct beam & $\begin{array}{l}\text { Peak power density at spot area: } 32 \mathrm{~kW} / \\
\mathrm{cm}^{2}\end{array}$ \\
\hline Energy distribution: Gaussian & $\begin{array}{l}\text { Average power density at spot area: } \\
6.4 \times 10^{-3} \mathrm{~W} / \mathrm{cm}^{2}\end{array}$ \\
\hline Peak power: $8 \mathrm{MW}$ & Beam divergence: $3 \times 1 \mathrm{mrad}^{2}$ \\
\hline Average power: $1.6 \mathrm{~W}$ & \\
\hline
\end{tabular}

mode, delivery system, energy distribution, peak power, average power, focal spot diameter, focus spot area, peak power density at spot area, average power density at spot area and beam divergence.

A modular Avantes spectrometer with $1 \mathrm{~ms}$ gate width and $0.5 \mathrm{~nm}$ spectral resolution over the wide UV/VIS/ NIR spectral range of $200-1100 \mathrm{~nm}$ was employed to record the successive fluorescence emission signals which were collected by a fiber bundle (FC-UV400-2-ME$\mathrm{SR})$. Figure 1a displays the perpendicular configuration of LIF spectroscopy using fiber optic interconnections. Subsequently, PL measurements were carried out by making use of JASCO FP-6200 spectrofluorometer with a spectral resolution of $1 \mathrm{~nm}$, which benefited from an excitation source of Xenon lamp. The detector was a high sensitive photomultiplier (PMT) to characterize the PL emissions. As a consequence, LIF employed a coherent light with better spectral resolution and flexible arrangement, whereas PL utilized an incoherent light source and a PMT detector. Despite the fact that PL suffered from lower spectral resolution, it benefited from higher sensitivity.

Furthermore, NIR measurements were implemented using the modular spectroscopy set-up, including an incoherent halogen lamp (HL-2000-HP) and an NIR spectrometer (NIRQuest) made by Ocean Optics. The nominal power of this incoherent source was $20 \mathrm{~W}$, while the typical output power delivered to the target through the optical fiber reached $\approx 9 \mathrm{~mW}$. In general, the high sensitive NIR spectrometer benefited from the InGaAs detector with an active wavelength ranging from 900 to $2500 \mathrm{~nm}$ and a $5 \mathrm{~nm}$ spectral resolution. Figure $1 \mathrm{~b}$ displays the chemical structures of LV, FU and Xeloda.

\section{Results and Discussion \\ LIF Spectra}

Figure 2a illustrates the UV-VIS absorption spectra in different concentrations of Xeloda. The measurements indicated the high absorbance at three characteristic

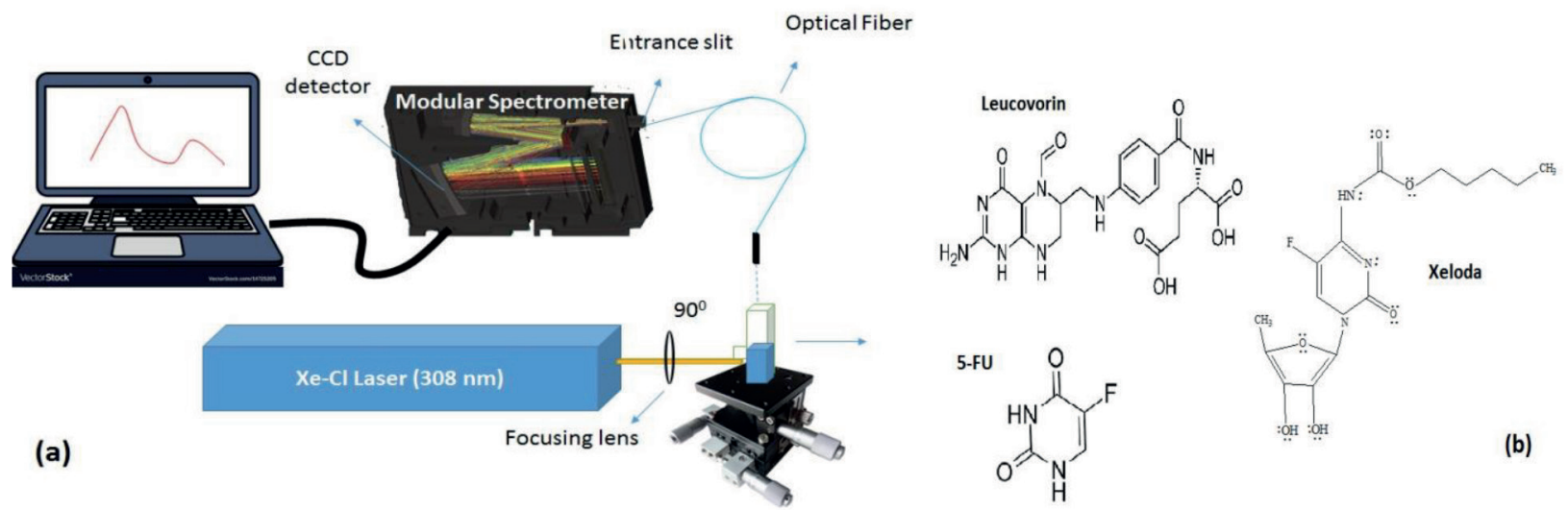

Figure 1. (a) Experimental configuration for LIF measurements of Xeloda, including the UV coherent source of the XeCl laser at 308 nm, CCD based modular spectrometer with Czerny-Turner configuration over UVIVIS wavelength range and the collecting optical fiber bundles (FC-UV400-2-ME-SR) (b) Chemical structures of Leucovorin, 5-FU and Xeloda compounds. 
maximum peaks of 216, 237 and $302 \mathrm{~nm}$ in the UV region. The absorbance demonstrates a linear relation in terms of dilute molar concentrations (less than 0.06 $\mathrm{mg} / \mathrm{mL}$ ) due to Beer-Lambert equation. Accordingly, Figure 2a-inset depicts the spectral absorbance versus various chemodrug concentrations emphasizing a linear behavior. Figure $2 \mathrm{~b}$ shows a number of LIF spectra in different Xeloda concentrations ranging from 0.001 to $10 \mathrm{mg} / \mathrm{mL}$. The maximum peak intensity was achieved for the characteristic concentration of $\mathrm{C}_{\mathrm{P} 1}=0.05 \mathrm{mg} / \mathrm{mL}$ at $\lambda_{\max }=410.5$. In fact, $\mathrm{C}_{\mathrm{P} 1}$ is a certain concentration of Xeloda where the maximum LIF intensity is observed. In pharmaceutical application, the parameter $\mathrm{C}_{\max }$ is defined as the maximum drug concentration in human plasma after characteristic time $t_{1 / 2}$. Here, $C_{P 1}$ lay within the allowable range of Xeloda concentrations at human plasma $\left(\mathrm{C}_{\max }\right)$ during the final stage of the metabolic process of chemotherapy administration. ${ }^{17,18}$ Furthermore, the fluorescence emission underwent weaker and red-shifted signals at larger chemodrug concentrations. Figure 3a displays LIF peak intensity versus different characteristic concentrations ranging between 0.001 and $1 \mathrm{mg} / \mathrm{mL}$ at $\lambda_{\text {exc }}=308 \mathrm{~nm}$. The fluorescence signal intensity elevated against Xeloda concentration below $\mathrm{C}_{\mathrm{P} 1} \approx 0.05 \mathrm{mg} / \mathrm{mL}$; however, it underwent a sharp drop to $\mathrm{C}_{\text {Xeloda }} \approx 0.2 \mathrm{mg} /$ $\mathrm{mL}$ corresponding to a minimal LIF signal. The largest
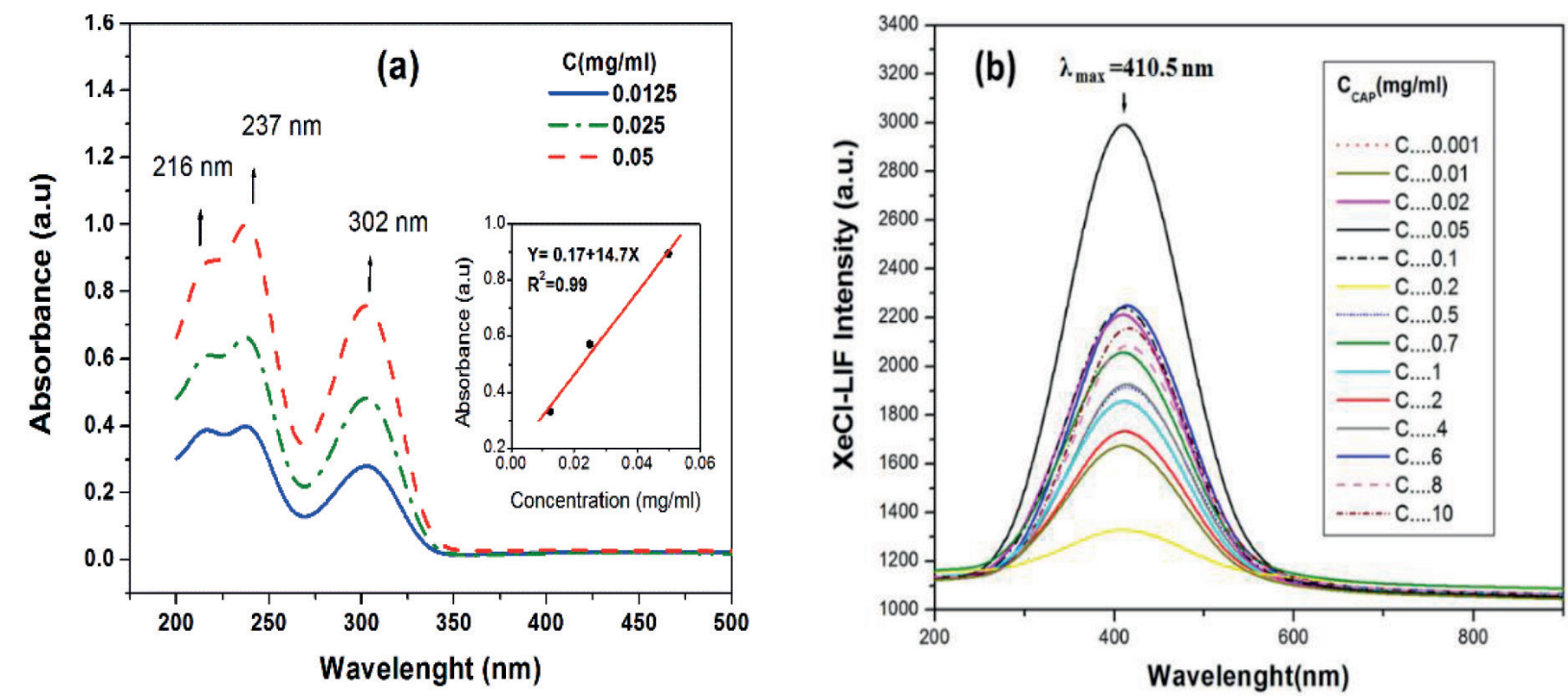

Figure 2. (a) UV-VIS absorption spectra of capecitabine in different concentrations characterizing three absorbance peaks at 216, 237 and $302 \mathrm{~nm}$. Inset: absorbance peak intensity versus fluorophore concentration (b) LIF spectra of different Xeloda concentrations ranging $0.001-10 \mathrm{mg} / \mathrm{mL}$.
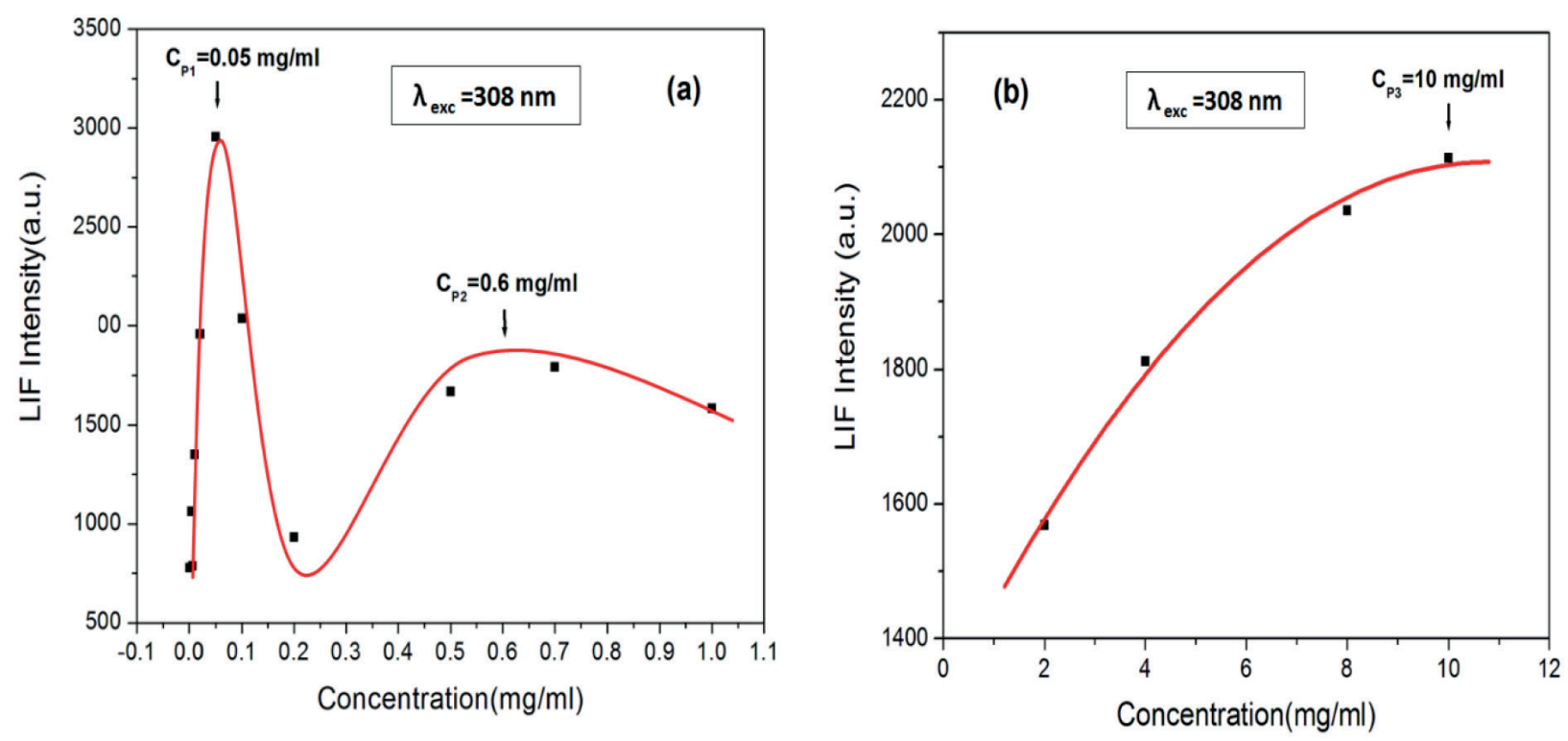

Figure 3. LIF Peak Intensity Versus Xeloda Concentration Ranging (a) $0.01-1 \mathrm{mg} / \mathrm{mL}$ (b) $1-10 \mathrm{mg} / \mathrm{mL}$. 
LIF signal was obtained at $\mathrm{C}_{\mathrm{p} 1}=0.05 \mathrm{mg} / \mathrm{mL}$, where there was another peak which took place at $\mathrm{C}_{\mathrm{P} 2}=0.6 \mathrm{mg} / \mathrm{mL}$. Figure $3 \mathrm{~b}$ illustrates the signal at larger concentrations ranging between 1 and $10 \mathrm{mg} / \mathrm{mL}$ with a third peak at $\mathrm{C}_{\mathrm{P} 3}=10 \mathrm{mg} / \mathrm{mL}$ accompanying a shifted wavelength of $\sim 6$ $\mathrm{nm}$. In general, three maximum peaks were observable at absorbance and fluorescence spectra. It is worth noting that Xeloda is a compound fluorophore in the form of $\mathrm{LV}+\mathrm{FU}$, whose therapeutic effect is notably enhanced, particularly for colon and breast metastases.

\section{PL Spectra}

The fluorescence properties of various blood antigens/ antibodies have previously been investigated using PL spectra. ${ }^{19}$ Furthermore, PL and LIF spectroscopies have recently been employed to study drug-protein interactions..$^{20,21}$ In the present study, fluorescence properties for different Xeloda concentrations were assessed using PL measurements. Figure 4a depicts the Xeloda fluorescence spectra for various concentrations ranging from 0.007 to $10 \mathrm{mg} / \mathrm{mL}$. PL measurements demonstrate the maximum signal intensity for the concentration of $\mathrm{C}_{\mathrm{P} 1}=0.05 \mathrm{mg} / \mathrm{mL}$ at $\lambda_{\max }=406 \mathrm{~nm}$. The fluorescence signal becomes faint and red-shifted at a larger range of chemodrug concentrations. Figure $4 \mathrm{~b}$ displays the variation of PL signal versus concentration ranging from 0.007 to $0.2 \mathrm{mg} / \mathrm{mL}$. Similar to LIF, maximum and minimum PL intensities appear at fluorophore concentrations of $\mathrm{C}_{\mathrm{P} 1}=0.05$ and $0.2 \mathrm{mg} / \mathrm{mL}$ respectively. Figure $4 \mathrm{c}$ plots the PL signal at dense solutions ranging from 1 to $10 \mathrm{mg} / \mathrm{mL}$ with a maximum signal at Xeloda concentration of $\mathrm{C}_{\mathrm{P} 2}=10 \mathrm{mg} / \mathrm{mL}$.

The higher concentrations emphasize the smooth rise of fluorescence intensity versus Xeloda concentration
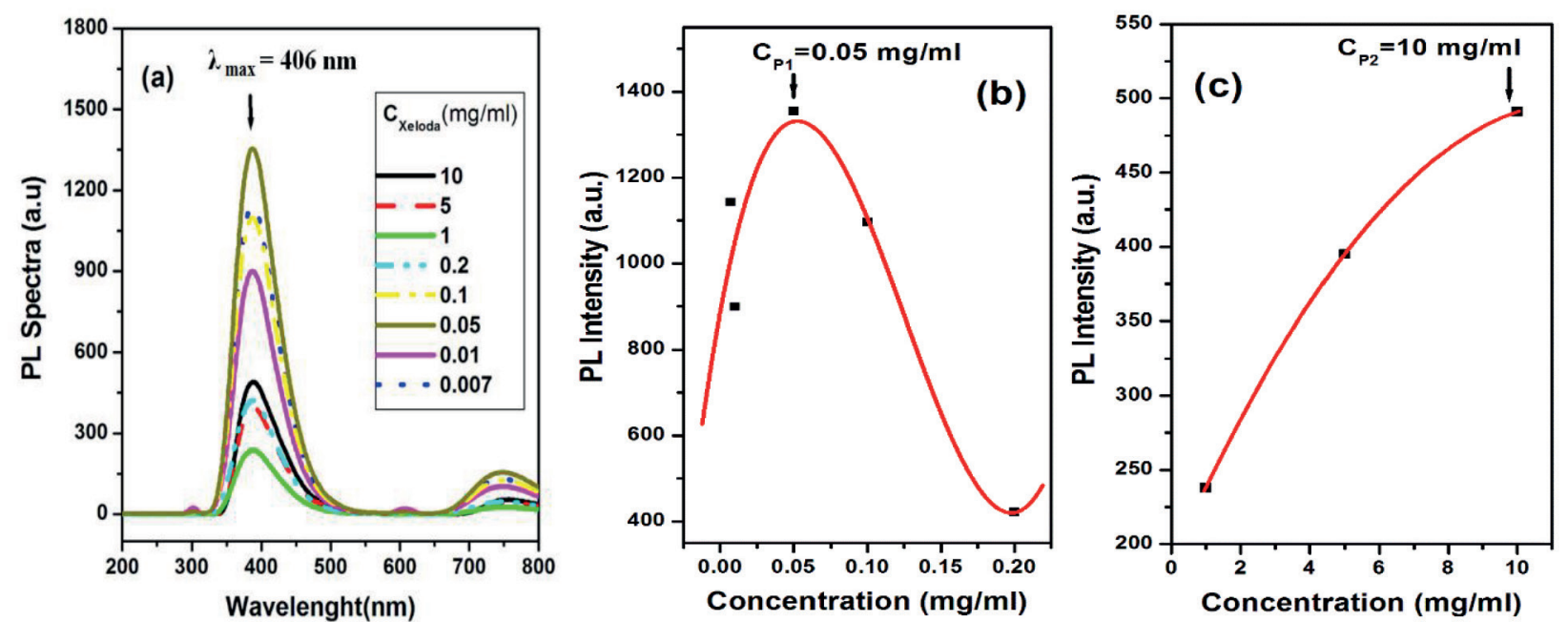

Figure 4. (a) PL spectra of different fluorophore concentrations ranging 1-10 mg/mL. Corresponding PL peak intensity versus Xeloda concentration ranging (b) $0.01-0.2 \mathrm{mg} / \mathrm{mL}$ (c) $1-10 \mathrm{mg} / \mathrm{mL}$.
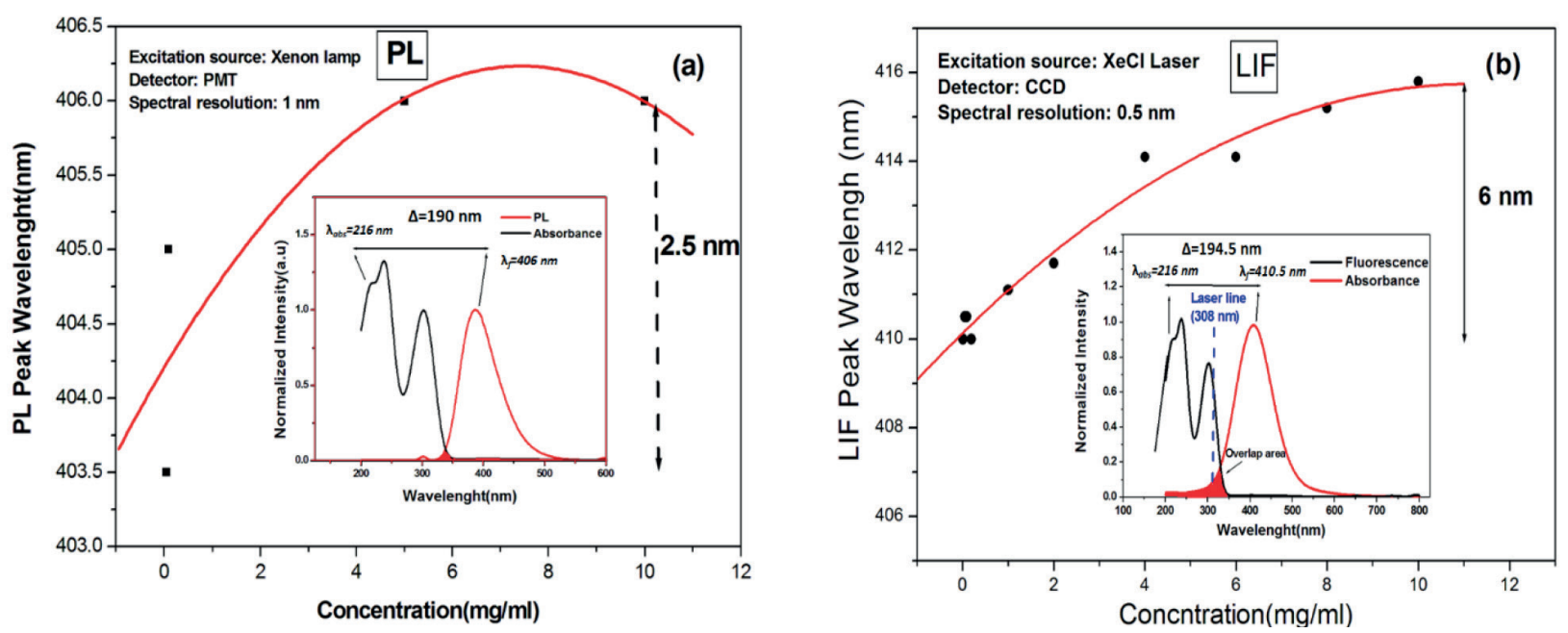

Figure 5. (a) Spectral red shift of PL peak intensity versus Xeloda concentration between 0.01-10 mg/mL. Inset: Normalized absorbance and PL spectra of Xeloda where the overlapping spectral area is highlighted (b) Spectral red shift of LIF peak intensity versus Xeloda concentration between $0.01-10 \mathrm{mg} / \mathrm{mL}$. Inset: Normalized absorbance and LIF spectra of Xeloda with the highlighted overlapping spectral area. 
ranging between 1 and $10 \mathrm{mg} / \mathrm{mL}$. It is worth noting that PL signals are in good agreement with data given by the LIF measurements. Fluorescence characteristic emission arises from the highest intermolecular transitions as well as the quenching effects, the formation of overwhelming dimmers and collisional dissipation.

\section{Red Shift in LIF and PL Spectra}

Recently, the behaviour of the spectral shift of LIF emission for different fluorophores has been reported. ${ }^{22,23}$ In this study, the spectral shifts of Xeloda fluorescence emission in LIF and PL spectra were analyzed. Figure 5a illustrates the peak emission wavelength of PL signals in terms of Xeloda concentration indicating a slight red shift of $2.5 \mathrm{~nm}$. Figure 5a-inset depicts the absorbanceemission spectra characterizing a pronounced overlap area during PL measurements. Figure 5b plots the variation of the peak wavelength of LIF spectra in terms of fluorophore concentration. Figure 5b-inset represents the overlap area between absorbance emission spectra in LIF measurements. The hatched area is highlighted to indicate large reabsorption effects during LIF measurements. Spectral overlap covers a larger area in LIF than PL spectra, leading to a notable red shift in LIF $(\sim 6 \mathrm{~nm})$ against PL experiments $(<2.5 \mathrm{~nm})$. This originates from the coherent and incoherent excitation sources at LIF against PL measurements. The incoherent Xenon lamp is used for PL spectroscopy, while a coherent excimer laser is employed to obtain LIF spectra. Some spectral characteristics of LIF and PL are listed in Table 2, including the excitation wavelength $\lambda_{\text {exc }}$, the excitation pulse width $\Delta \lambda_{\text {exc }}$, emission wavelength $\lambda_{f}$, FWHM of emission peak $\Delta \lambda_{f}$ and spectral/stokes shifts.

Here, spectroscopic characteristics of Xeloda as a common chemodrug were studied to verify the feasible medical applications of light interaction with this medication during simultaneous chemotherapy. LIF emission as a simple technique could be employed to monitor the chemodrug distribution and its cytotoxicity. This introduced a novel LIF-based protocol for simultaneous diagnosis and treatment of cancer.

\section{NIR Spectra}

NIR spectroscopy is a common technique for the identification of different tablets. ${ }^{24}$ Here, this method was employed to study the structure of chemical bindings in different Xeloda concentrations in deionized water over a wide spectral range of 900-2500 nm. Figure $6 a$ depicts the NIR spectra for different concentrations from 0.01 to $10 \mathrm{mg} / \mathrm{mL}$. Figure $6 \mathrm{a}$ highlights the formation of molecular bands at a certain Xeloda concentration of $10 \mathrm{mg} / \mathrm{mL}$. Although there was no covalent bonding for concentrations below $0.2 \mathrm{mg} / \mathrm{mL}$, NIR absorbance was elevated at dense solutions. The appearance of several sharp peaks emphasizes the formation of strong intermolecular bindings such as the first overtone of

Table 2. Spectral Properties of LIF and PL Spectra of Xeloda

\begin{tabular}{ccccccc}
\hline & $\boldsymbol{\lambda}_{\text {exc }}(\mathbf{n m})$ & $\Delta \boldsymbol{\lambda}_{\text {exc }}(\mathbf{n m})$ & $\boldsymbol{\lambda}_{\mathbf{f}}(\mathbf{n m})$ & $\Delta \boldsymbol{\lambda}_{\mathbf{f}}(\mathbf{n m})$ & Spectral Shift $(\mathbf{n m})$ & Stokes Shift $(\mathbf{n m})$ \\
\hline Xeloda-LIF & 308 & 2.5 & 406 & 130 & 6 & 194.5 \\
Xeloda-PL & 300 & 6 & 410 & 118 & 2.5 & 190 \\
\hline
\end{tabular}
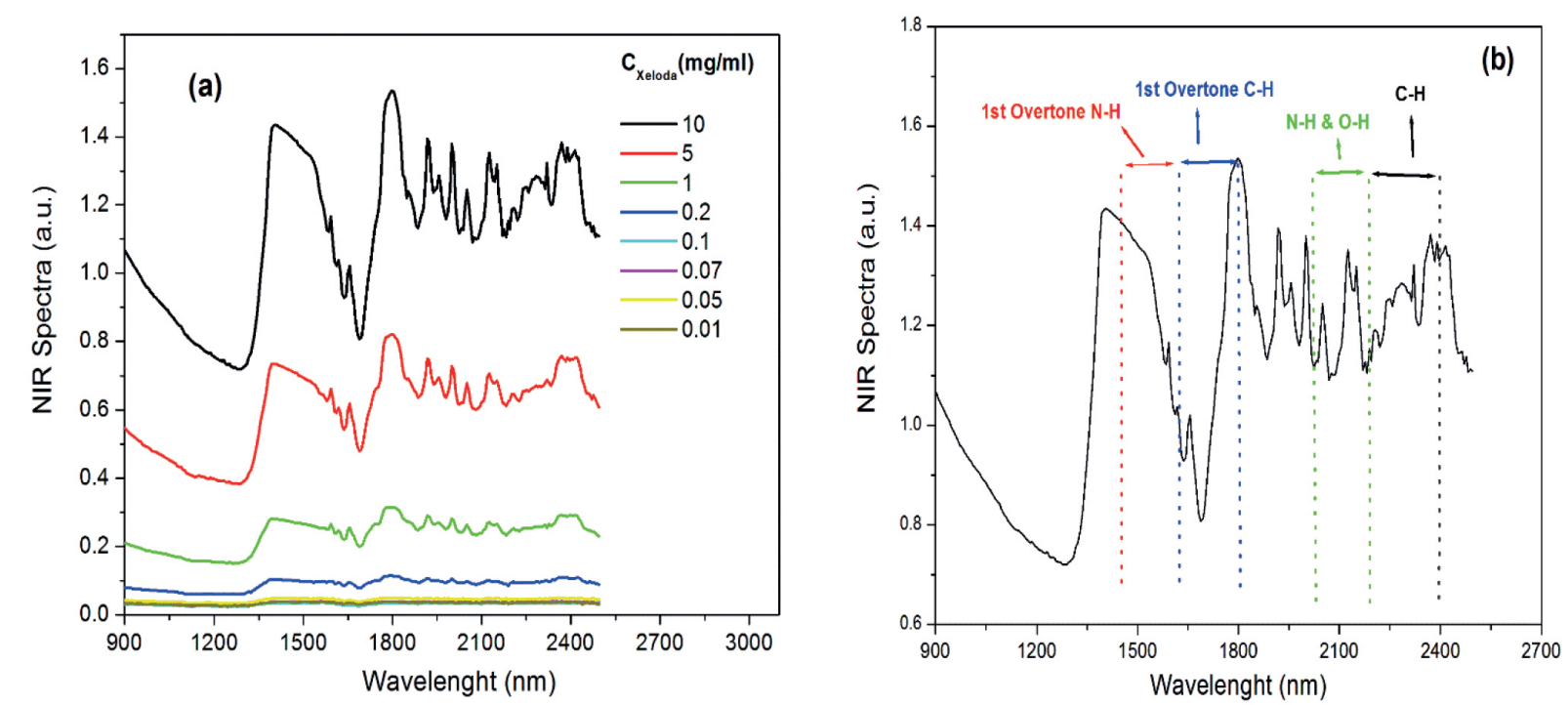

Figure 6. (a) NIR spectra for different concentrations of Xeloda ranging $0.01-10 \mathrm{mg} / \mathrm{mL}$. (b) The formation of intermolecular $\mathrm{NH}, \mathrm{CH}$ and $\mathrm{OH}$ bonds at $10 \mathrm{mg} / \mathrm{mL}$ concentration of Xeloda in deionized water dilution. Note that no bond appears below $0.2 \mathrm{mg} / \mathrm{mL}$ concentrations, while new $\mathrm{NH}, \mathrm{OH}$ and $\mathrm{CH}$ bindings are formed at larger concentrations. 
$\mathrm{C}-\mathrm{H}, \mathrm{N}-\mathrm{H}$ and $\mathrm{O}-\mathrm{H}$. The new intermolecular bonds were formed at dense solutions, including $\mathrm{NH}(1400-1500 \mathrm{~nm})$, $\mathrm{CH}$ (1400-1500 and 2200-2400 nm) and $\mathrm{OH}(2000-2300$ $\mathrm{nm})$. It is necessary to mention that $\mathrm{NH}$ and $\mathrm{OH}$ bindings were overlapped over 2000-2300 nm.

Furthermore, these findings attest that the largest fluorescence intensity takes place at $\mathrm{C}_{\mathrm{P} 1}=0.05 \mathrm{mg} / \mathrm{mL}$, which is in agreement with the data given in Figure $3 \mathrm{a}$. At concentrations smaller than $0.2 \mathrm{mg} / \mathrm{mL}$ and larger than $0.05 \mathrm{mg} / \mathrm{mL}$, strong quenching occurs due to fluorescence energy transfer. ${ }^{25}$ On the other hand, new bindings are pronounced above $0.2 \mathrm{mg} / \mathrm{mL}$ to avoid excessive quenching events in favor of radiative emission. In fact, the major portion of the kinetic energy of species is imparted to the formation of molecular bindings. Consequently, the population of molecular transitions increases instead of non-radiative deactivations, leading to larger fluorescence signals according to Figure $3 b^{26,27}$

\section{Conclusion}

Xeloda as the oral chemodrug exhibits outstanding fluorescence properties over the maximum serum concentration regarding the pharmacokinetic variable of $\mathrm{C}_{\max }$. This characteristic provides physicians with advantages for the forthcoming LIF-based/in-vivo diagnosis during chemotherapy treatment. There are three typical absorbance peaks for Xeloda in the UV region; therefore, an appropriate excitation wavelength at $308 \mathrm{~nm}$ is selected and fluorescence emissions, as well as the corresponding spectral red shifts, are recorded. LIF measurements are performed by making use of the $\mathrm{XeCl}$ laser and CCD-based spectrometer. A maximum LIF signal is observed at $\mathrm{C}_{\mathrm{P} 1} \approx 0.05 \mathrm{mg} / \mathrm{mL}$ at $\lambda_{\max }=410.5$. PL signals are in good agreement with the data given by the LIF measurements. NIR spectra represent the chemical structure of Xeloda in different concentrations. In fact, for the concentrations above $0.2 \mathrm{mg} / \mathrm{mL}$, the population of molecular transitions is elevated according to the formations of new bindings, leading to the enhancements of fluorescence emissions. The corresponding spectral red-shift of $\sim 6 \mathrm{~nm}$ attests to the dominant reabsorption events at the allowable medical concentration. Xeloda distribution throughout the target tumor envisages a novel cancer diagnostic method based on its certain fluorescence properties.

This work enables us to carry out simultaneous diagnosis/imaging and treatment of cancerous tumors utilizing the UV coherent light alongside LIF spectroscopy. The excimer laser enables us to detect and ablate unhealthy tissues at the same time. The spectroscopic properties of Xeloda were investigated here to collect necessary data for biomedical engineers and clinicians who intend to carry out the clinical practice. LIF measurements of Xeloda as a biocompatible chemodrug/fluorophore using the $\mathrm{XeCl}$ laser could be suitable alternatives for the diagnosis and treatment of deep lesions via fiber in the near future.

\section{Ethical Considerations}

Not applicable.

\section{Conflict of Interests}

The authors declare no conflict of interest.

\section{Acknowledgments}

We are thankful to Mr. Shahriar Abolhosseini from Nuclear Science and Technology Research Institute, Tehran, Iran, for his collaborations.

\section{References}

1. Walko CM, \& Lindley C. Xeloda: a review. Clin Ther. 2015; 27(1): 23-44. doi: 10.1016/j.clinthera.2005.01.005

2. Masuda N, Lee SJ, Ohtani S, Im YH, Lee ES, et al. Adjuvant capecitabine for breast cancer after preoperative chemotherapy. N Engl J Med. 2017; 376(22): 2147-2159. doi: 10.1056/NEJMoa1612645

3. Di Costanzo F, Ravasio R, Sobrero A, Bertetto O, Vinante O, Luppi G, Labianca R, Amadori D, Barone C, Merlano $\mathrm{MC}$ and Longo F. Capecitabine versus bolus fluorouracil plus leucovorin (folinic acid) as adjuvant chemotherapy for patients with Dukes' C colon cancer. Clin Drug Investig. 2008; 28(10): 645-65. doi: 10.2165/00044011-20082810000005

4. Cunningham D, Starling N, Rao S, Iveson T, Nicolson M, Coxon F, et al. Capecitabine and oxaliplatin for advanced esophagogastric cancer. N Engl J Med. 2008; 358(1): 36-46. doi: 10.1056/NEJMoa073149

5. Rehse SJ, Salimnia H, Miziolek AW. Laser-induced breakdown spectroscopy (LIBS): an overview of recent progress and future potential for biomedical applications. J Med Eng Technol. 2012; 36(2):77-89. doi:10.3109/030919 02.2011 .645946

6. Treado PJ, Nelson MP, inventors; ChemImage Corp, assignee. Method for Raman chemical imaging of endogenous chemicals to reveal tissue lesion boundaries in tissue. United States patent US 6,965,793. 2005 Nov 15.

7. Ghasemi F, Parvin P, Motlagh NS, Amjadi A, Abachi S. Laser induced breakdown spectroscopy and acoustic response techniques to discriminate healthy and cancerous breast tissues. Appl Opt. 2016; 55(29): 8227-35. doi: 10.1364/AO.55.008227

8. Bahreini M. Role of optical spectroscopic methods in neuro-oncological sciences. J Lasers Med Sci. 2015; 6(2):51.

9. Ghasemi F, Parvin P, Motlagh NS, Abachi S. LIF spectroscopy of stained malignant breast tissues. Biomed Opt Express.2017; 8(2): 512-23. doi:10.1364/BOE.8.000512

10. Liu W, Zhang X, Liu K, Zhang S, Duan Y. Laser-induced fluorescence: Progress and prospective for in vivo cancer diagnosis. Chin Sci Bull. 2013;58(17): 2003-2016. doi: 10.1007/s11434-013-5826-y

11. Motlagh NH, Parvin P, Ghasemi F, Atyabi F, Jelvani S, 
Abolhosseini S. Laser induced fluorescence spectroscopy of chemo-drugs as biocompatible fluorophores: irinotecan, gemcitabine and navelbine. Laser Phys Lett 2016; 13(7):075604. https://iopscience.iop.org/ article/10.1088/1612-2011/13/7/075604/meta

12. Motlagh NS, Parvin P, Refahizadeh M, Bavali A. Fluorescence properties of doxorubicin coupled carbon nanocarriers. Appl Opt. 2017; 56(26):7498-503. doi: 10.1364/AO.56.007498

13. Motlagh NS, Parvin P, Ghasemi F, Atyabi F. Fluorescence properties of several chemotherapy drugs: doxorubicin, paclitaxel and bleomycin. Biomed Opt Express. 2016;7(6):2400-6. doi: 10.1364/BOE.7.002400

14. Pahang F, Parvin P, Ghafoori-Fard H, Bavali A, Moafi A. Fluorescence properties of methylene blue molecules coupled with metal oxide nanoparticles. OSA Continuum. 2020;3(3):688-97. doi:10.1364/OSAC.387557

15. Motlagh NH, Parvin P, Mirzaie ZH, Karimi R, Sanderson $\mathrm{JH}$, Atyabi F. Synergistic performance of triggered drug release and photothermal therapy of MCF7 cells based on laser activated PEGylated GO+ DOX. Biomed Opt Express. 2020;11(7): 3783-94. doi: 10.1364/BOE.389261

16. Jahdi SA, Parvin P, Seyedi S, Jelvani S. ArF laser induced fluorescence spectroscopy of the Capecitabine chemodrug. OSA Continuum. 2020;3(6):1477-89. doi: 10.1364/ OSAC. 384267

17. Reigner B, Verweij J, Dirix L, Cassidy J, Twelves C, Allman D, Weidekamm E, Roos B, Banken L, Utoh M, Osterwalder B. Effect of food on the pharmacokinetics of capecitabine and its metabolites following oral administration in cancer patients. Clin Cancer Res. 1998; 4(4):941-8.

18. Albanell J, Montagut C, Jones ET, Pronk L, Mellado B, Beech J, et al. A phase I study of the safety and pharmacokinetics of the combination of pertuzumab (rhuMab 2C4) and capecitabine in patients with advanced solid tumors. Clin
Cancer Res. 2008; 14(9):2726-31. doi: 10.1158/1078-0432. CCR-07-1980

19. Seyedi SS, Parvin P, Jafargholi A, Hashemi N, Tabatabaee SM, Abbasian A, Khorrami A. Spectroscopic properties of various blood antigens/antibodies. Biomed Opt Express.2020; 11(4): 2298-312. doi: 10.1364/BOE.387112

20. Seyedi S, Parvin P, Jafargholi A, Jelvani S, Shahabi M, Shahbazi M, et al. Fluorescence properties of Phycocyanin and Phycocyanin-human serum albumin complex. Spectrochim Acta a Mol Biomol Spectrosc. 2020;118468. doi:10.1016/j.saa.2020.118468

21. Lakowicz JR, editor. Principles of fluorescence spectroscopy. Springer US; 2013; Apr 17.

22. Bavali A, Parvin P, Mortazavi SZ, Mohammadian M, Pour MM. Red/blue spectral shifts of laser-induced fluorescence emission due to different nanoparticle suspensions in various dye solutions. Appl Opt. 2014; 53(24): 5398-409. doi:10.1364/AO.53.005398

23. Bavali A, Parvin P, Tavassoli M, Mohebbifar MR. Angular distribution of laser-induced fluorescence emission of active dyes in scattering media. Appl Opt. 2018; 57(7): B328. doi: 10.1364/AO.57.000B32

24. Ulmschneider M, Penigault E. Non-invasive confirmation of the identity of tablets by near-infrared spectroscopy. Analysis. 2000; 28(4): 336-46. doi:10.1051/analusis:2000124

25. Gather M C, Yun, S. H. Bio-optimized energy transfer in densely packed fluorescent protein enables near-maximal luminescence and solid-state lasers. Nat Commun. 2014; 5(1): 1-8. doi:10.1038/ncomms6722

26. Mardani Kh, Parvin P, Bavali A, Moafi A, Ehtesham A. Angular study of laser-induced fluorescence emission of hybrid media based on Stern-Volmer formalism. OSA Continuum. 2021; 4(1):15-29. doi:10.1364/OSAC.408779

27. B. Wardle. Principles and applications of photochemistry. John Wiley \& Sons; 2009. 